\title{
Planificación energética para el ahorro de fueloil en una lavandería industrial
}

\author{
Energy planning for fuel oil saving in an industrial laundry
}

\author{
José A. Madrigal $^{1} \quad$ Juan J. Cabello Eras ${ }^{2 *} \quad$ Hernán Hernández Herrera $^{2}$ \\ Vladimir Sousa Santos ${ }^{2} \quad$ Milén Balbis Morejón ${ }^{2}$ \\ Recibido 22 de abril de 2015, aceptado 21 de noviembre de 2016 \\ Received: April 22, $2015 \quad$ Accepted: November 21, 2016
}

\begin{abstract}
RESUMEN
En el presente trabajo se diseña y aplica un procedimiento de planificación energética, compatible con la norma ISO 50001, a una lavandería industrial. Se analizan tres variables de entrada: nivel de producción, estado técnico del sistema y régimen de operación. Se examina el pasado, presente y futuro del consumo del fuel oíl en el sistema de generación y uso del vapor, estableciendo las causas del deterioro del indicador de consumo. A través de un estudio de carga y capacidad en el sistema se identificaron las potencialidades de ahorro mejorando el esquema de operación. Se propuso un nuevo esquema para la operación de las calderas el cual fue implementado lográndose un ahorro del 16\% de combustible. Con los resultados de las mediciones se elabora la línea base y se establecen nuevas metas.
\end{abstract}

Palabras clave: Ahorro de combustible, indicador de consumo, ISO 50001, planificación energética, calderas.

\begin{abstract}
In this paper was applied a method of energy planning, compatible with ISO 50001 standard, in an industrial laundry. In the analysis, three input variables were considered: level of production, technical condition of the system and operating regime. The past, present and future of fuel oil consumption in the steam generation and use was examined, establishing the causes of deterioration of consumption indicator. A study of load and capacity in the system identified potential savings through improved operating regime. A new regime for the operation of boilers was proposed and implemented, saving $16 \%$ of fuel. With the results of these measurements, the baseline was developed and goals are established.
\end{abstract}

Keywords: Fuel saving, consumption indicator, ISO 50001, energy planning, boilers.

\section{INTRODUCCIÓN}

El turismo es uno de los sectores con mayor crecimiento en la economía mundial. La Organización Mundial del Turismo estima un crecimiento anual del 3,3\% para el periodo 2010-2030 [1] y se conoce que tiene una contribución de alrededor del $5 \%$ en las emisiones globales de dióxido de carbono [2].

En la industria del turismo el sub-sector hospedaje es uno de los principales consumidores energéticos, debido a que demanda grandes cantidades de

1 Facultad de Ingeniería Mecánica. Universidad de Cienfuegos. Carretera a Rodas Km 4, Cuatro Caminos. Cienfuegos, Cuba. CP 59430. E-mail: jamadrigal@ucf.edu.cu

2 Grupo de Investigación en Optimización Energética GIOPEN. Facultad de Ingeniería. Universidad de la Costa CUC. Calle 58 \#55-66. CP 080006. Barranquilla. Colombia. hhernand16@cuc.edu.co; mbalbis1@cuc.edu.co; jcabello2@cuc.edu.co; vsousa1@cuc.edu.co

* Autor de correspondencia 
energía eléctrica, así como, vapor y agua caliente en las habitaciones para asegurar el confort de los huéspedes [3-6], estas dos últimas obtenidas en las calderas las cuales funcionan generalmente con fuel oíl o gas [7].

También tienen altos consumos en actividades auxiliares que ocasionalmente están integradas a su esquema operacional; en otros es frecuente encontrar que algunos servicios como: lavandería, elaboración de alimentos, restaurante, tienda y seguridad son subcontratados [8].

La Gestión Energética (GE) en la industria hotelera ha alcanzado buenos resultados en muchos países; en la literatura especializada se recogen varias experiencias [9-13], sin embargo, no es frecuente encontrar reportes acerca de la GE en los servicios auxiliares y especialmente en las lavanderías.

En Cuba se han logrado avances significativos en la Eficiencia Energética (EE) [14-18 a través del Programa de Ahorro Energético de Cuba (PAEC), que ha permitido el ahorro de más de 8 millones de toneladas de petróleo equivalente desde 2005 [19], con un impacto significativo en la dimensión económica, social y energético ambiental del país lo que resulta en que Cuba es uno de los países de América Latina con mejor relación entre la Huella Ecológica y específicamente la de carbono y el Índice de Desarrollo Humano, logrando un incremento del Producto Interno Bruto en los últimos años manteniendo prácticamente constantes estos indicadores.

Sin embargo a nivel corporativo no existe el nivel deseado en la GE [20-21]. El sector turístico no es ajeno a esto y aunque la mayoría de los hoteles han implementado Sistemas de Gestión Ambiental, las acciones dirigidas a disminuir los consumos de energía se han enfocado principalmente a acciones aisladas de EE [22]. En las pequeñas empresas que brindan servicios auxiliares a los hoteles la aplicación de la GE es más baja aún.

El ahorro de energía se ha convertido en un tema de vital importancia tanto para las autoridades, como para las empresas, en su búsqueda del aumento de la competitividad y las mejoras del desempeño ambiental. Esto se debe a su estrecha relación con el cambio climático y la degradación ambiental [23]. Muchos países han acrecentado sus esfuerzos para estandarizar la gestión de la energía como una forma de mejorar continuamente la Eficiencia Energética. Los Estados Unidos con la norma ANSI/MSE 2000:2008"A Management System for Energy, Dinamarca con la DS 2403:2001 "Energy Management-Specification", Japón con la JIS Z 9211 y 9212, Canadá con la PLUS 1140, y Alemania con la VDI 4602 fueron punteros en este proceso. Asimismo, la Comunidad Europea elaboró el Marco de Acción en Materia de Clima y Energía hasta el año 2030 y la Organización Internacional de Normalización (ISO) aprobó la norma ISO 50001 "Sistemas de gestión de la energía. Requisitos con orientación para su uso".

En Cuba, las actividades relacionadas con la ISO 50001 apenas han comenzado, la Oficina Nacional de Normalización la ha adoptado para su uso en el país como NC ISO 50001 desde al año 2012; pero ninguna empresa nacional ha sido certificada.

El presente trabajo se centra en el estudio y reducción del consumo de combustible en una lavandería industrial, que presta servicio a hoteles en Cuba, apoyados para ello en un procedimiento para la planificación en el marco de la norma ISO 50001.

\section{MATERIALES Y MÉTODOS}

La GE se basa en la aplicación de herramientas técnicas y organizativas basadas en la aplicación de los principios y procedimientos de la gestión total de la calidad y se orienta a lograr una mejora continua en el desempeño energético de las empresas [24].

En el año 2011 con la aprobación de la ISO 50001 se da un paso trascendental en el desarrollo de la GE, al disponerse de un estándar internacional que define los requisitos generales de un Sistema de Gestión de la Energía (SGE), para establecer una política energética con objetivos concretos encaminados a mejorar la EE. La aplicación de esta norma permite definir las áreas que más influyen sobre el consumo energético, elaborar pronósticos del consumo de energía, identificar potenciales de ahorro y proponer las acciones necesarias para su aprovechamiento. Hasta marzo del 2014 casi 7000 empresas a nivel mundial habían certificado su SGE por ISO 50001 [25]. 
La norma plantea un modelo de cuatro etapas: política energética, planeación energética, implementación y verificación, todas insertadas en un ciclo de mejora continua [26].

La etapa de planificación energética y sus componentes se consideran el núcleo de un SGE, ya que proporcionan las bases para el desarrollo de las estrategias de mejora del desempeño energético. Esta consta de tres etapas [27-28]; en la Figura 1 se pueden apreciar aplicadas a un sistema de generación y uso del vapor.

\section{Lavanderías}

Las lavanderías desde el punto de vista energético se dividen en dos áreas, generación y consumo de vapor. En el área de generación se produce el vapor mediante las calderas, la cuales generalmente operan con diésel, fuel oúl o gas. En el área de consumo de vapor se encuentran los equipos que realizan la tarea principal de la instalación que son el lavado, secado y planchado de la lencería. Los portadores energéticos principales que se consumen en las lavanderías son la electricidad y el combustible para la generación del vapor.

Para la evaluación de la eficiencia productiva de las lavanderías se utilizan varios indicadores [28];

Productividad $=\frac{\text { kgrl }}{\text { Nro trab }}$

Donde:

kgrl- Kilogramos de ropa lavada

Nro trab-Número de trabajadores
Rentabilidad $=\frac{G T}{I T}$

Donde:

GT- Gastos Totales.

IT- Insumos Totales.

Índice de consumo $=\frac{C I}{k g r l}$

Donde:

CI - Consumo de insumos.

La EE se controla a través de los índices de consumo.

\section{Lavandería unicornio}

La lavandería Unicornio se encuentra instalada en la ciudad de Cienfuegos, Cuba y es la encargada de lavar la lencería de 6 Hoteles de turismo internacional de la provincia. Tiene una capacidad máxima instalada para $200.000 \mathrm{~kg}$ mensuales de lencería procesada, aunque la producción real se encuentra en el orden de los $150.000 \mathrm{~kg}$. La instalación cuenta con dos calderas que poseen una capacidad de 4.000 y $2.000 \mathrm{~kg} / \mathrm{h}$ de vapor respectivamente.

Como equipamiento tecnológico cuenta con nueve lavadoras, ocho secadoras y dos máquinas planchadoras de diferentes capacidades.

En la Figura 2 se muestra la estructura de consumo de los portadores energéticos en la lavandería durante el año 2013 expresados en toneladas de petróleo equivalente (TPE). En esta se puede apreciar que el fuel oíl representa más del $70 \%$ del consumo energético de la entidad.

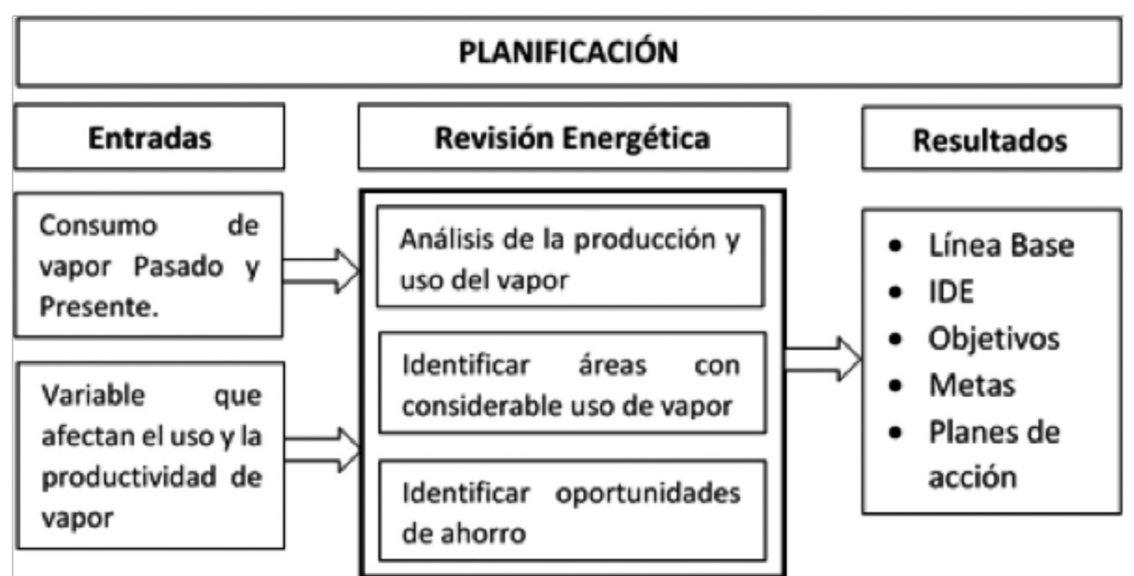

Figura 1. Etapas de la Planificación Energética en un sistema de vapor. 


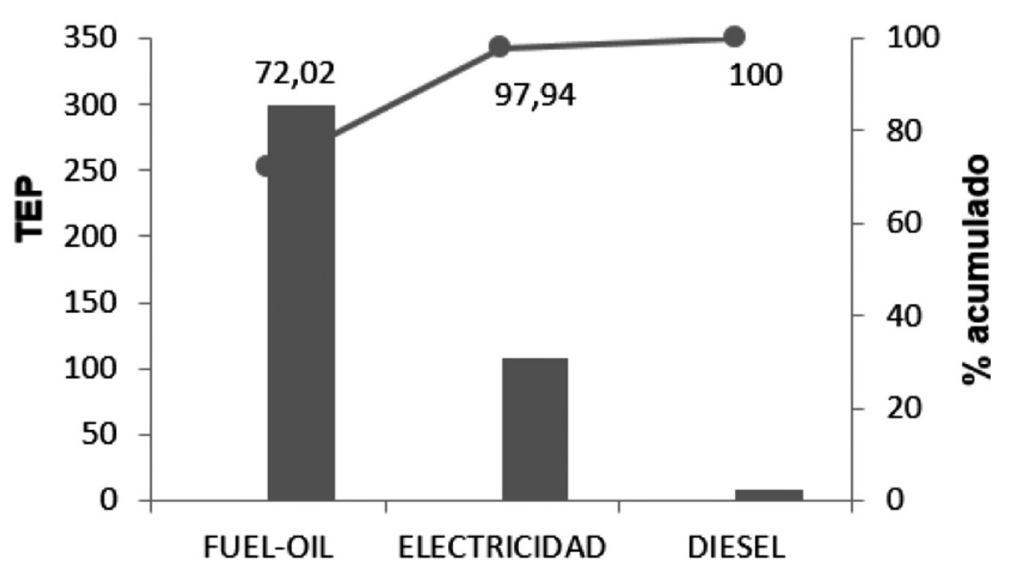

Figura 2. Estructura de consumo de portadores energéticos en el año 2013.

\section{APLICACIÓN DEL PROCEDIMIENTO DE PLANIFICACIÓN ENERGÉTICA A LA GENERACIÓN Y USO DEL VAPOR}

\section{Definición de las entradas}

Para la definición de las entradas se estudió el flujo productivo y consumo de la instalación durante los años 2012 y 2013, también se aplicaron técnicas de trabajo en grupo, con operadores y el personal técnico encargado de la operación y mantenimiento, a partir de las cuales se identificaron tres variables de entrada: nivel de producción, estado técnico del sistema y régimen de operación.

\section{Nivel de producción}

En la Figura 3 se muestra el comportamiento de la producción y el consumo de combustible en el año 2013, observándose que existe una estrecha relación entre ambos y que el nivel de producción de la lavandería depende de la temporada turística, la cual es mayor en los meses de temporada alta, durante el invierno, y menor en la baja turística, durante el verano.

En la Figura 4 se muestra el comportamiento del indicador de consumo de fuel oíl en el año 2013. El Ministerio del turismo en Cuba (MINTUR) establece como indicador los litros de combustible consumido entre los kilogramos de lencería procesada $(\mathrm{L} / \mathrm{kg})$, para el mismo establece como valor máximo 0,23 $\mathrm{L} / \mathrm{kg}$ de lencería procesada [29]. En el gráfico se aprecia claramente una tendencia al deterioro del indicador en los últimos meses del año lo que dio origen al presente trabajo.

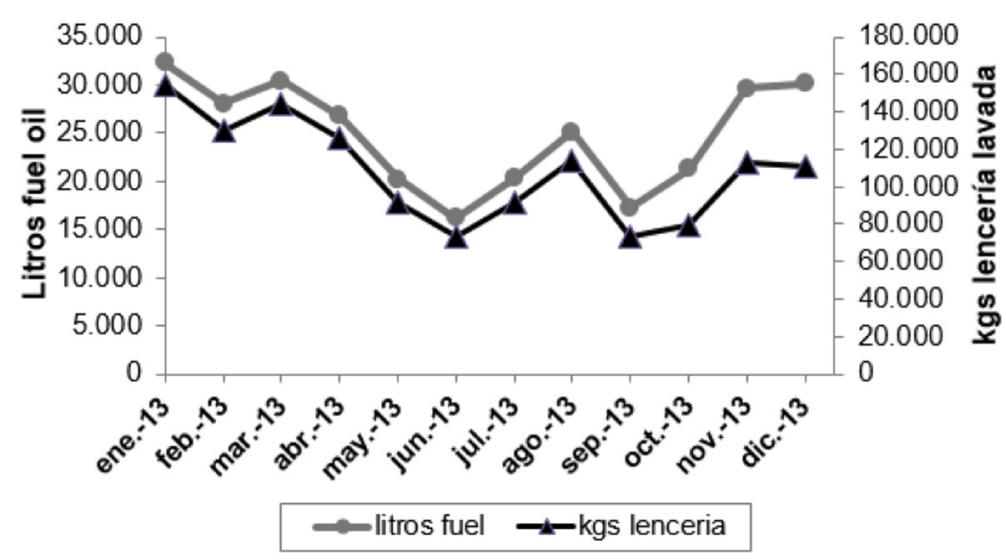

Figura 3. Comportamiento de la producción y el consumo de combustible en el año 2013. 


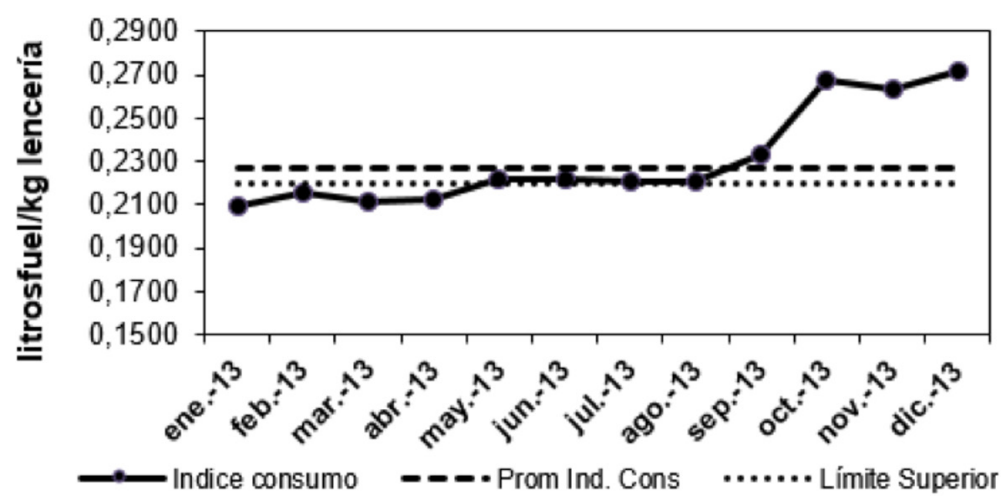

Figura 4. Control del índice de consumo de combustible en el periodo analizado.

La pertinencia de Índice de Consumo se evaluó a través de un análisis de correlación entre el consumo mensual de fuel-oíl y la cantidad de lencería procesada en los años 2012 y 2013, el coeficiente de correlación $\left(\mathrm{R}^{2}\right)$ fue de 0,86 lo que representa un resultado satisfactorio Figura 5 a). Un análisis similar se realizó excluyendo los meses en los que el índice tuvo un comportamiento anormal y $\mathrm{R}^{2}$ mejoró hasta un valor de 0,96 , como se observa en la Figura 5 b), Esto demuestra que el índice de consumo puede utilizarse como Indicador de Desempeño Energético [30-31].

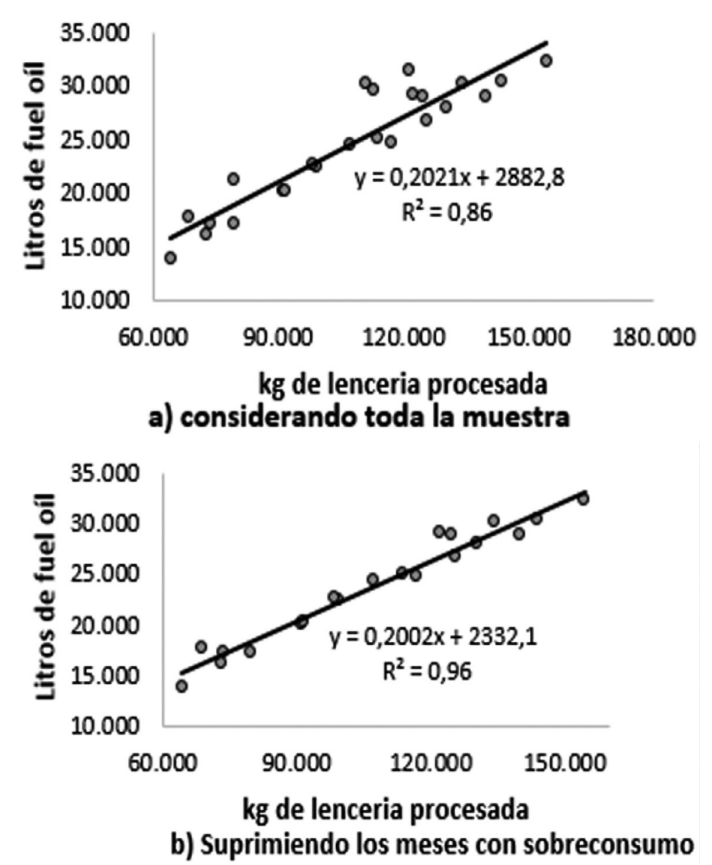

Figura 5. Diagrama de correlación del consumo fuel oíl vs producción.
En la Figura 6 se muestra el comportamiento del Índice de Consumo contra la producción, determinándose que por debajo de la producción crítica de la empresa que es de $90.000 \mathrm{~kg} / \mathrm{mes}$, el índice comienza a deteriorarse.

\section{Revisión energética}

Por medio de la revisión energética se determina el desempeño energético de la organización. Una parte esencial de ella es la evaluación del desempeño energético de la organización para establecer una línea base e identificar acciones que la mejoren. Para realizar la evaluación se utilizaron los datos estadísticos de consumo de combustible, producción, los manuales de los equipos, el estado técnico de los equipos de generación y uso del vapor, la realización de mediciones en tiempo real para el balance de vapor. También se desarrolló una evaluación en planta que incluyó una sesión de trabajo en grupo con el personal para identificar las prácticas operacionales y sus tiempos de ejecución.

\section{Estado técnico del sistema de generación y uso del vapor}

Para la evaluación del estado técnico del sistema se analizaron los registros de mantenimiento y se realizó una inspección en toda la instalación estableciendo que:

- Hay una tendencia al deterioro acelerado en el aislamiento del sistema de vapor.

- A partir del mes de Octubre del 2013 se registraron fugas de vapor en las trampas y no han sido corregidas.

- Existen notables pérdidas en el tanque de retorno de condensado.

- Hay fugas de vapor en los consumidores finales. 


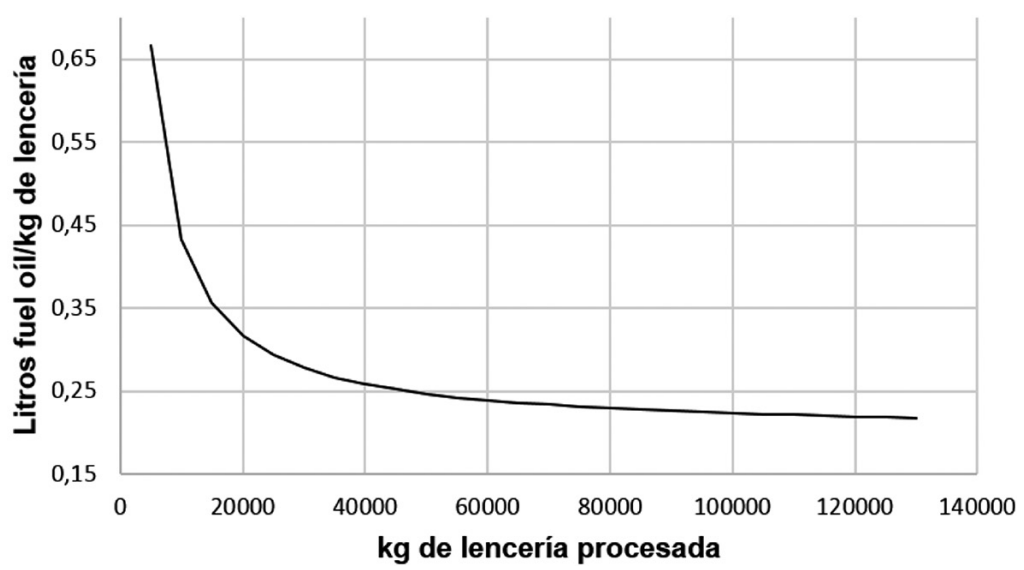

Figura 6. Comportamiento del índice de consumo contra producción.

Con este análisis se concluye que el deterioro marcado de las instalaciones de transporte de vapor es causa visible del comportamiento del indicador de consumo en los meses finales del año 2013; las acciones de mantenimiento y reparación realizadas en enero del 2014 orientaron el indicador a valores adecuados.

\section{Estrategia operacional}

En la temporada de alza turística la lavandería procesa entre 100.000 y $150.000 \mathrm{~kg}$ de lencería mensual, equivalente a procesar entre 5.000 y $7.000 \mathrm{~kg}$ diarios. Mientras que en la temporada de baja se procesa entre 60.000 y $100.000 \mathrm{~kg}$, trabajando en ocasiones por debajo de la condición crítica de $90.000 \mathrm{~kg} / \mathrm{mes}$.

El esquema de operación para la producción de vapor en el procesamiento de la lencería es el mismo en ambas temporadas y se muestra en la Figura 7; en este se encienden ambas calderas comenzando por la de $4.000 \mathrm{~kg} / \mathrm{h}$ y en las horas que la demanda, en función de las condiciones de diseño lo justifique,

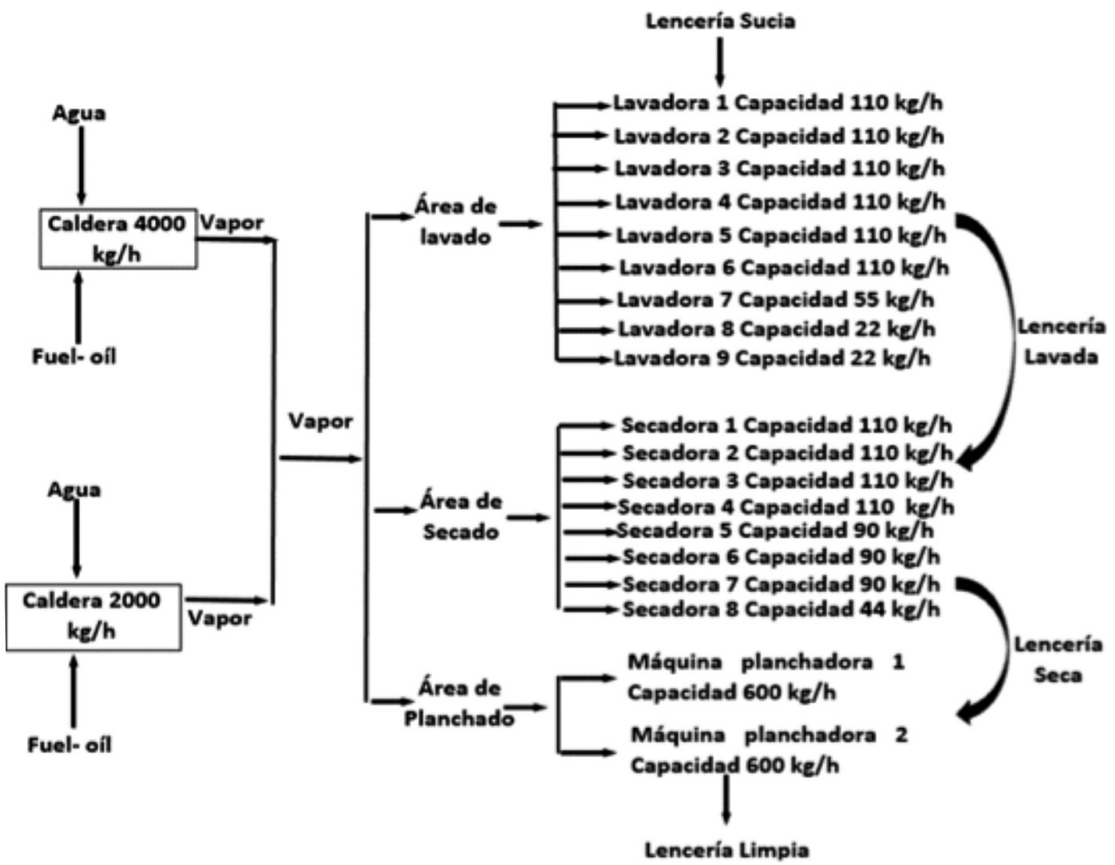

Figura 7. Esquema de operación para el procesamiento de la lencería. 
se incorpora la caldera de $2.000 \mathrm{~kg} / \mathrm{h}$. Según las condiciones de diseño de los equipos instalados la demanda máxima es de $4.492 \mathrm{~kg} / \mathrm{h}$ como se observa en la Tabla 1.

\section{Balance al consumo de vapor de proceso}

Como primer paso del balance se midió el consumo real a plena capacidad durante 15 días. En la Tabla 1 se pueden apreciar el promedio de estas mediciones.

De la Tabla 1 se puede concluir que hay varios equipos sobredimensionados, particularmente las lavadoras tienen una demanda real de $320,6 \mathrm{~kg} / \mathrm{h}$ y una capacidad nominal de $1.742 \mathrm{~kg} / \mathrm{h}$.

Junto al balance se realizó un censo de carga y una evaluación del trabajo de los equipos, donde se pudo detectar que las dos máquinas planchadoras se mantenían trabajando constantemente durante todo el proceso y no a máxima capacidad, lo que ocasionaba un sobreconsumo de vapor.

Se realizó un acomodo de los kilogramos de lencería procesada de la manera mostrada en la Figura 8, lo que permitió que los equipos trabajen a máxima capacidad y eficiencia, disminuyendo el sobreconsumo de vapor en un $6,4 \%$ diario.

A partir de este esquema de carga se desarrolló un programa de mediciones para determinar el comportamiento de la demanda real de vapor por hora en un día en que se trabaja a máxima capacidad, procesándose $7.000 \mathrm{~kg}$ de lencería. En la Figura $9 \mathrm{se}$ muestra el resultado observándose que la demanda comienza a aumentar a partir de las 6 am y el pico se produce entre 10 y 11 am con un aprovechamiento de la capacidad de generación instalada (ACI) del 44,3\%.

Como conclusión del balance se plantea que no es necesario utilizar simultáneamente ambas calderas, ya que la de $4.000 \mathrm{~kg} / \mathrm{h}$ satisface la demanda real en un $155 \%$.

Se efectuaron mediciones durante la operación de la lavandería en la caldera de $4.000 \mathrm{~kg} / \mathrm{h}$ para determinar el factor de carga $\phi$ en el horario de demanda máxima.

$$
\varnothing=\frac{T_{t q}}{T_{t g v}}
$$

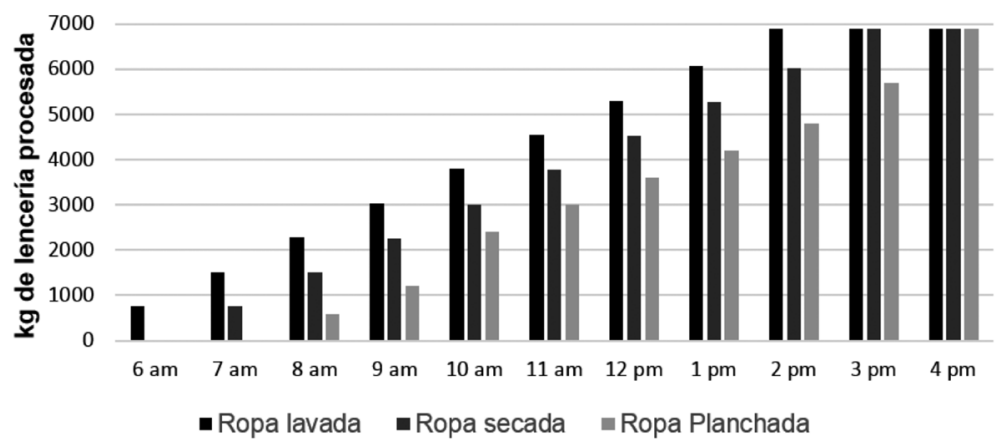

Figura 8. Esquema de producción con reacomodo de kg de lencería.

Tabla 1. Consumo real de vapor por equipos a máxima demanda.

\begin{tabular}{|l|c|c|c|}
\hline \multicolumn{1}{|c|}{ Equipos } & Cantidad & Capacidad kg/h & Demanda de vapor kg/h según datos Chapa \\
\hline Máquinas planchadoras & 2 & 600 & 350 \\
\hline Secadoras & 4 & 110 & 250 \\
\hline Secadoras & 3 & 90 & 300 \\
\hline Secadoras & 1 & 44 & 150 \\
\hline Lavadoras & 6 & 110 & 248 \\
\hline Lavadoras & 1 & 55 & 124 \\
\hline Lavadoras & 2 & 22 & 65 \\
\hline
\end{tabular}




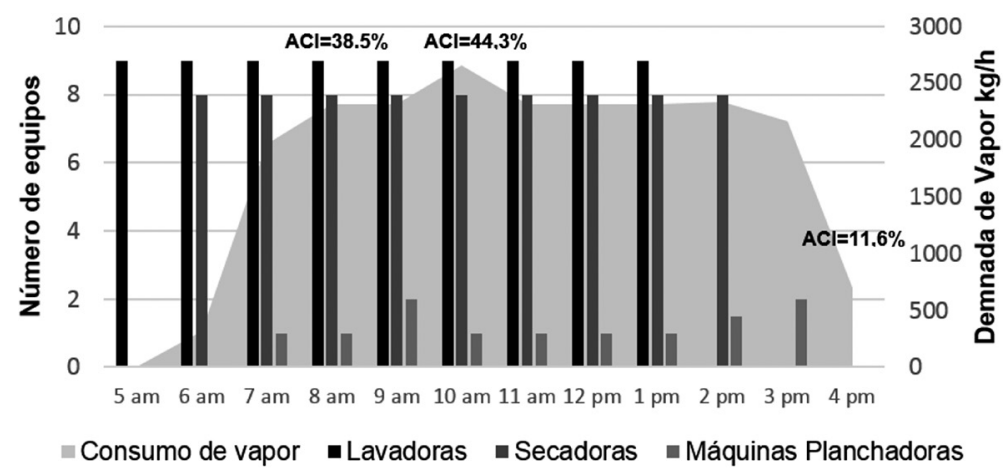

Figura 9. Comportamiento de la demanda de vapor para $7.000 \mathrm{~kg}$ de lencería procesada.

Donde:

$T_{t q}:$ es el tiempo de trabajo quemador en horas.

$T_{g v}$ : es el tiempo de trabajo del generador de vapor en horas.

El factor de carga promedio de la caldera fue de 0,58 lo que confirma su alto grado de sobredimensionamiento.

Se realizó un ajuste del horario de utilización de la capacidad instalada como se muestra en la Figura 10, de forma tal que la demanda máxima no supere $\operatorname{los} 2.000 \mathrm{~kg} / \mathrm{h}$ de vapor. Bajo estas condiciones se puede utilizar la caldera más pequeña con un alto coeficiente de aprovechamiento y menor consumo. Las evaluaciones realizadas demostraron que esto es posible siempre que la cantidad de lencería que se procesa sea menor de $5.300 \mathrm{~kg}$ diarios.

En la Figura 11 se muestran los resultados de las mediciones en la demanda y producción bajo estas condiciones.

\section{RESULTADOS Y DISCUSIÓN}

A partir de los resultados anteriores se modificó el esquema de generación de la lavandería en el año 2014 a través del ajuste en el uso de las calderas. Para los días en que se procesaron más de $5.300 \mathrm{~kg}$ de lencería, se puso en funcionamiento la caldera de $4.000 \mathrm{~kg} / \mathrm{h}$, se realizaron mediciones y bajo estas condiciones el factor de carga medio de la caldera alcanzó el valor de 0,84 , lo que trajo consigo una mejora del índice de consumo alcanzándose un valor promedio de $0,195 \mathrm{~L} / \mathrm{kg}$ y una reducción del consumo de fuel oíl del $15 \%$ respecto al esquema operativo tradicional.

Para los días donde se procesó menos de $5.300 \mathrm{~kg}$ se utilizó solamente la caldera de $2.000 \mathrm{~kg} / \mathrm{h}$. El factor de carga $\phi$ de la caldera fue del $96 \%$ y el índice de consumo se redujo hasta $0,185 \mathrm{~L} / \mathrm{kg}$ lo que representó una reducción del $20 \%$ en el consumo de fuel oíl respecto al esquema operativo

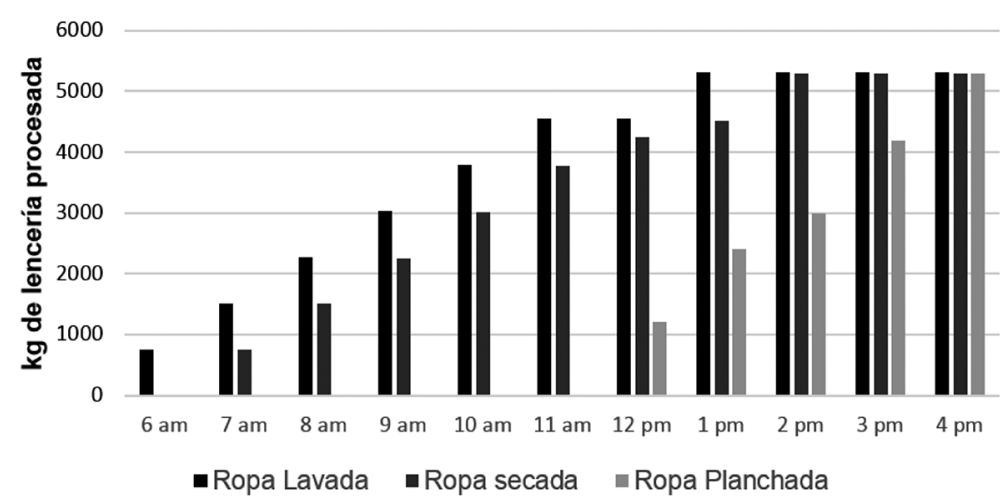

Figura 10. Esquema de producción con reacomodo de carga para $5.300 \mathrm{~kg}$ lencería procesada. 


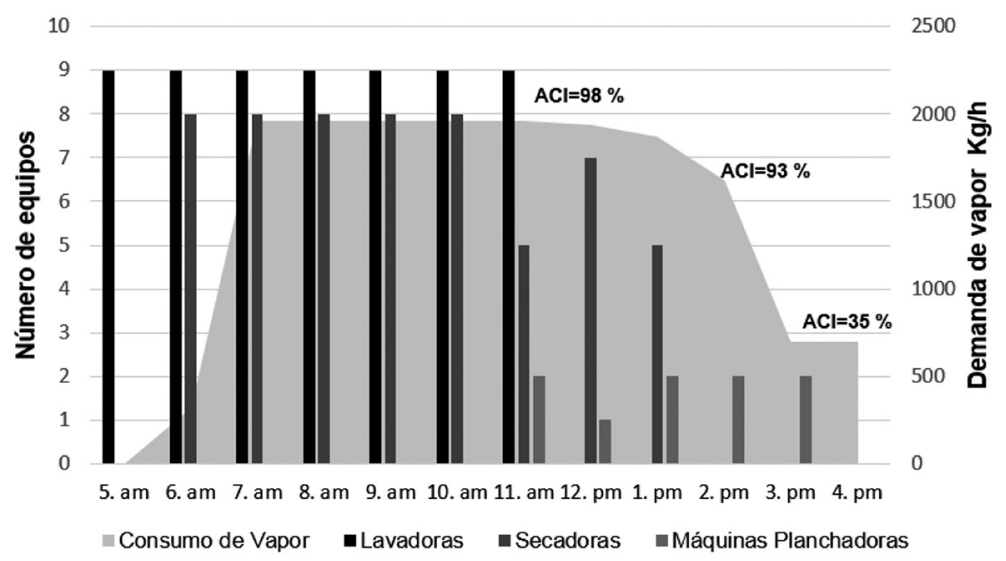

Figura 11. Comportamiento de la demanda de vapor para $5.300 \mathrm{~kg}$ de lencería procesada.

tradicional. Debido a la aplicación de estas medidas el ahorro anual en el 2014 respecto al año anterior fue de 47.672 litros lo que representa un $16 \%$ de ahorro.

Con los resultados de las mediciones en el año 2014 se elaboró el diagrama de consumo contra producción mostrado en la Figura 12, este diagrama permite obtener la línea base de consumo para este año; a partir de esta y tomando los mejores valores de consumo contra producción durante el año 2014 se puede establecer la línea meta para el año 2015. De esta forma se puede predecir, que si en el año 2015 se logra un comportamiento como los mejores meses del 2014 la empresa podrá lograr un ahorro de 770 Litros fuel oíl mensual.

\section{CONCLUSIONES}

La aplicación de un procedimiento para la planificación energética en la lavandería Unicornio permitió:

- Identificar las causas del deterioro del indicador de consumo de fuel oíl.

- Identificar las fuentes de ineficiencias en el proceso ocasionadas por el sobredimensionamiento de los equipos y a un esquema de operación de los generadores de vapor deficiente.

- Se propuso e implementó una nueva estrategia operacional de las calderas para el caso de procesar más de $5.300 \mathrm{~kg}$ de lencería que permite un ahorro del 14\%, entrando en operación la caldera de $4.000 \mathrm{~kg} / \mathrm{h}$. Para el caso de que la

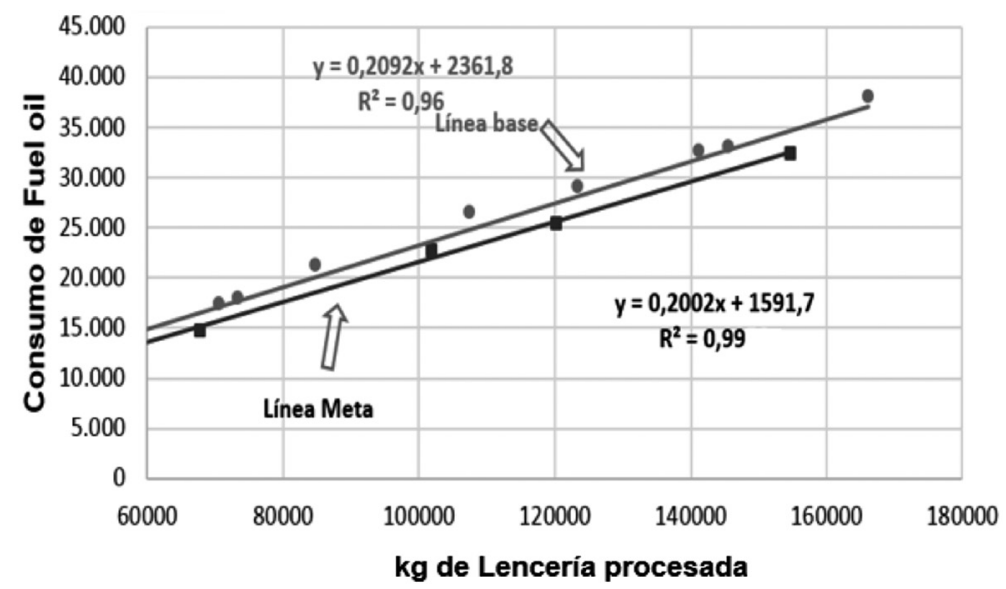

Figura 12. Línea Base Energética y Línea Energética Meta. 
producción planificada sea menor de $5.300 \mathrm{~kg}$ se propuso el uso exclusivo de la caldera de $2000 \mathrm{~kg} / \mathrm{h}$ con un potencial de ahorro del $20 \%$. Esto significó un ahorro de 47.672 litros de fuel oíl en el año 2014 lo que representa un 16\%.

- Se obtuvo la línea base del año 2014 con el nuevo esquema de operación y se propone la línea meta para el año 2015 con un ahorro de 9.240 litros anuales.

\section{REFERENCIAS}

[1] United Nations World Tourism Organization. "UNWTO tourism highlights 2014 edition", pp. 1-2. Fecha de actualización Diciembre del 2013. Consultado en Noviembre del 2014. URL: http://mkt.unwto.org/es/publication/ panorama-omt-del-turismo-internacionaledicion-2014

[2] D. Scott, B. Amelung, S. Becken, J. Ceron, G. Dubois, S. Gössling and M. Simpson. "Climate change and tourism: Responding to global challenges". 2nd International Conference on Climate Change and Tourism. Davos, Switzerland. 1-4 Octubre, 2008. URL: http://sdt.unwto.org/sites/all/files/docpdf/ climate2008.pdf

[3] M. Best and B. Thapa. "Motives, facilitators and constraints of environmental management in the Caribbean accommodations sector". Journal of Cleaner Production. Vol. 52, pp. 165-175. Agosto 2013. ISSN: 0959-6526.

[4] F. Silva, A. López and C. J. Renedo. "El uso de humidificadores como alternativa energética al aire acondicionado: caso de un edificio docente en Santiago de Compostela". Revista Facultad de Ingeniería. Vol. 64, pp. 22-31. Septiembre 2012. ISSN: 0120-6230.

[5] D. Shiming and J. Burnett. "Energy use and management in hotels in Hong Kong”. International Journal of Hospitality Management. Vol. $21 \mathrm{~N}^{\circ}$ 4, pp. 371-380. December, 2002. ISSN 0278-4319.

[6] J. Vergara, V. Quesada and I. Blanco. "Análisis de la calidad en el servicio y satisfacción de los usuarios en dos hoteles cinco estrellas de la ciudad de Cartagena (Colombia) mediante un modelo de ecuaciones estructurales". Ingeniare. Revista chilena de ingeniería. Vol. $19 \mathrm{~N}^{\circ} 3$, pp. 420-428. Septiembre 2011. ISSN 0718-3305.
[7] Agencia Valenciana de Energía. "Guía de ahorro y Eficiencia Energética en Establecimientos Hoteleros de la Comunidad Valenciana", pp. 28-32. Fecha de actualización: Julio 2014. Consultado en Agosto del 2014. URL: http://www.magrama.gob.es/es/ cambio-climatico/planes-y-estrategias/ GuiaEficienciaHoteles_CV_tcm7-293295. pdf

[8] D. Shi-Ming and J. Burnett. "A study of energy performance of hotel buildings in Hong Kong”. Energy and Buildings. Vol. $31, \mathrm{~N}^{\circ} 1$. pp. 7-12. January, 2000. ISSN: 0378-7788.

[9] A. Milojkovic, M. Nikolic and A. Stankovic. "Improvement of energy efficiency in hospitality-towards sustainable hotel". IV International Symposium for Students of Doctoral Studies in the Fields of Civil Engineering, Architecture and Environmental Protection. Nis. Serbia y Montenegro. 21-22 de Septiembre 2012.

[10] R. Priyadarsini, W. Xuchao and L. Eang. "A study on energy performance of hotel buildings in Singapore". Energy and Buildings. Vol. $41 \mathrm{~N}^{\mathrm{o}} 12$, pp. 1319-1324. December, 2009. ISSN: 0378-7788.

[11] M. Hui and M. Wong. "Benchmarking the energy performance of hotel buildings in Hong Kong". Liaoning Hong Kong Joint Symposium. Dalian, China. 2-3 de Julio 2010.

[12] J. Chun Wang. "A study on the energy performance of hotel buildings in Taiwan". Energy and Buildings. Vol. 49, pp 268-275. June, 2012. ISSN: 0378-7788.

[13] S. Deng. "Energy and water uses and their performance explanatory indicators in hotels in Hong Kong". Energy and Buildings. Vol. 35 $\mathrm{N}^{\circ} 8$, pp. 775-784. September, 2003. ISSN: 0378-7788.

[14] International Atomic Energy Agency. "Cuba: A Country Profile on Sustainable Energy Development", pp. 79-106. Fecha de actualización Agosto 2008. Fecha de Consulta: Octubre 2014. URL: http:// www-pub.iaea.org/mtcd/ publications/pdf/ pub1328_web.pdf

[15] D. Perez and I. Lopez. "Evaluation of energy policy in Cuba using ISED. Natural Resources Forum". Vol. $29 \mathrm{~N}^{\circ}$ 4. pp 298-307. 2005. DOI: 10.1111/j.14 778947.2005.00142.x. 
[16] J. Cabello, A. Sagastume, D. García, J. Cogollos, L. Hens and C. Vandecasteele. "Bridging universities and industry through cleaner production activities. Experiences from the Cleaner Production Center at the University of Cienfuegos, Cuba". Journal of Cleaner Production. Vol. 108, pp. 873-882, December 2015. ISSN: 0959-6526.

[17] J. Suárez, P. Beatón, R. Faxas and O. Pérez. "Energy, environment and development in Cuba". Renewable and Sustainable Energy Reviews. Vol. 16, N 5. pp. 2724-2731. Junio 2012. ISSN 1364-0321.

[18] Carpio, C. Coviello, M. Horta, L. Peña, J. Gamarra, A. "Situación y perspectivas de la eficiencia energética en América Latina y el Caribe". http://www.cepal.org/es/ publicaciones/3726-situacion-perspectivasla-eficiencia-energetica-america-latinacaribe-situation. pp. 97-109, Octubre 2009, Consultado Septiembre 2016.

[19] Cabello, J. Garcia, Sagastume, A. Priego, $\mathrm{R}$ and Vandecasteele, C. "An approach to sustainable development the case of Cuba". Environment, Development and Sustainability. Vol. $14 \mathrm{~N}^{\circ} 4$, pp. 573-59. Febrero. 2012. ISSN 1387-585X.

[20] A. Borroto, M. Lapido, J. Monteagudo, M. Armas, M. Montesinos, J. Delgado, A. Padrón, P. Viego and F. González. "La gestión energética: una alternativa eficaz para mejorar la competitividad empresarial". Energética. Vol. 33, pp. 65-69. Julio 2005. ISSN 0120-9833.

[21] M. Käkönen, H. Kaisti and J. Luukkanen. "Energy revolution in Cuba: pioneering for the future". pp. 13-19. Fecha de actualización abril 2014. Consultado en Octubre 2014. URL: http://www.utu.fi/fi/yksikot/ffrc/julkaisut/etutu/Documents/eBook_4-2014.pdf.

[22] R. Silva, R. Perdomo and E. González, "Use of more efficient lamps in the Breezes Varadero Hotel". Retos Turísticos. Vol. 12, $\mathrm{N}^{\circ}$ 3. pp. 12-22. Mayo 2013. ISSN 2224-7947.

[23] S. Mínguez, A. Díaz, M. García y F. Silva. "Modelo de cálculo de las emisiones difusas de gases de efecto invernadero procedentes del transporte. Análisis según variables de diseño urbanístico". Revista Facultad de Ingeniería, $\mathrm{N}^{\circ}$ 73. pp. 200-213. Diciembre 2014. ISSN 0120-6230.
[24] A. Borroto and J. Monteagudo. "Gestión y Economía Energética". Editorial Universidad de Cienfuegos, Vol. 1, No 104 pp. 6-16. Cienfuegos, Cuba. ISBN: 959-257-114-7. 2006.

[25] Organización de las Naciones Unidas para el Desarrollo Industrial (ONUDI). 2014. "Actividades de la ONUDI relacionadas con la energía Informe del Director General". Disponible en: http://www.unido.org/ fileadmin/user_media/PMO/IDB/IDB42 / idb42_13s.pdf.

[26] J. Soto, A. Borroto, M. Alpha, R. González, M. Curvelo and A. Diaz. "Diseño y aplicación de un procedimiento para la planificación energética según la NC-ISO 50001:2011”. Ingeniería Energética. Vol. $35 \mathrm{~N}^{\circ}$ 1, pp. 38-47. Enero 2014. ISSN $1815-5901$.

[27] A. Borroto. "Recomendaciones Metodológicas para la Implementación de Sistemas de Gestión de la Energía según la Norma ISO 50001". Editorial Universo Sur. 1era edición. pp. 12-27. Cienfuegos, Cuba. ISBN 978959-257-360-4. 2013.

[28] E. Saumel and O. Parada. "La eficiencia y calidad del servicio en el proceso de lavandería tintorería en el hotel Meliá de Santiago de Cuba". Observatorio de la Economía Latinoamericana. $\mathrm{N}^{\circ} 130$. Abril 2010. ISSN 1696-8352.

[29] I. Camargo, R. Ucio and R. Rodríguez. "Sistema de indicadores para evaluar el desempeño en el hotel Los Jazmines". Avances. Vol. 14, $\mathrm{N}^{\circ}$ 4, pp. 1-11. Octubre 2012. ISSN 1562-3297.

[30] W. Pistoia, B.Van Rietbergen, E. Lochmüller, C. Lill, F. Eckstein and P. Rüegsegger. "Estimation of distal radius failure load with micro-finite element analysis models based on three-dimensional peripheral quantitative computed tomography images". Bone. Vol. $30 \mathrm{~N}^{\circ} 6$, pp. 842-848. Junio 2002. ISSN: 8756-3282.

[31] R. Martínez, R. Boueri y M. Escalona. "Correlación entre la evaporación en tina y la evapotranspiración de referencia en cinco estaciones climatológicas de Venezuela". Bioagro. Vol. $17 \mathrm{~N}^{\circ}$ 2, pp. 79-83. Abril 2005. ISSN: 1316-3361. 